\title{
Complications of airway self-expandable metallic stent in benign airway diseases
}

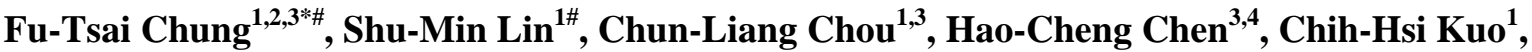 \\ Horng-Chyuan Lin ${ }^{1}$, Chien-Ying Liu ${ }^{1}$, Chun-Hua Wang ${ }^{1}$, Han-Pin Kuo ${ }^{1}$, Chih-Teng Yu ${ }^{1 *}$ \\ ${ }^{1}$ Department of Thoracic Medicine, Chang Gung Memorial Hospital, College of Medicine, Chang Gung University, Taipei, Taiwan \\ ${ }^{2}$ Graduate Institute of Clinical Medical Sciences, College of Medicine, Chang Gung University, Taoyuan, Taiwan \\ ${ }^{3}$ Department of Thoracic Medicine, Saint Paul's Hospital, Taoyuan, Taiwan \\ ${ }^{4}$ Department of Thoracic Medicine, Shuang Ho Hospital, Taipei Medical University, Taipei, Taiwan \\ Email: *vikingchung@gmail.com
}

Received 5 November 2012; revised 17 December 2012; accepted 14 January 2013

\begin{abstract}
Objectives: To present the complications of Ultraflex Self-Expandable Metallic Stents (SEMSs) applied in patients with benign tracheobronchial diseases. Methods: Eighty patients received 124 SEMSs were retrospectively reviewed in a tertiary hospital. Results: The baseline characteristics of patients received SEMS implantation including age (mean + standard deviation: $62.5+14.8$ years), gender (male, $60.5 \%$ ), smoking $(\mathbf{4 6 . 8 \%})$, forced expiratory volume in first one second (FEV1) (mean + standard deviation, $0.92+$ $0.41 \mathrm{~L} / \mathrm{s})$, follow-up days after SEMS implantation (median (interquartile range); 457 (131 - 897)) and covered SEMS (33.9\%) were listed. Symptoms improved after SEMS implantation among 95 patients (76.6\%). Time to complications developed was 236 (median; interquartile range, 59 - 672) days. The overall complication rate was $41.9 \%$ after SEMS implantation. The complications included SEMS migration (6.5\%), granuloma (19.3\%) and SEMS fracture (16.1\%). Successful management rates of SEMS migration, granuloma and SEMS fracture were up to $100 \%, 83.3 \%$, and $85 \%$ respectively. Conclusions: Patients received SEMS implantation due to benign conditions had poor lung function and were old. The complication rate in patients with benign conditions was high after longer follow-up period, however, successful management achieved in most patients with complications.
\end{abstract}

Keywords: Ultraflex Self-Expandable Metallic Stent; Benign Airway Disease; Complication; Management

\footnotetext{
"Corresponding authors.

"Drs. F.-T. Chung and S.-M. Lin equally contribute to this article as first authors. None of the authors have any conflicts of interest to disclose.
}

\section{INTRODUCTION}

Patients with symptomatic central airway obstruction could be managed in the past ten years [1-3]. Interventional bronchoscopic techniques have been widely applied in patients with central airway lesions because of the advances in endobronchial stents and insertion skills [4-7]. Bronchoscopy with rigid or flexible bronchoscope is the common procedure of stent placement in those patients. However, some patients are not suitable to receive surgical intervention with rigid bronchoscope with a general anesthetic, due to illness severity and comorbidities. Self-Expandable Metallic Stents (SEMSs) can be successfully inserted using a flexible bronchoscope while the patient receives conscious sedation and a local anesthetic [8-10]. For some patients who are not eligible for surgical procedures or rigid bronchoscopy, self-expandable metallic stents (SEMSs) insertion offers a chance to relieve their symptoms $[10,11]$.

Despite SEMSs could relieve central airway obstruction in large tracheobronchial diseases, some complications such as migration, granuloma formation, stent fracture, recurrent lumen obstruction of the stent and increased bacterial colonization and impaired mucocilliary clearance were reported [10,12-14]. The United States Food and Drug Administration (FDA) has warned that SEMS implantation should be considered only if the patient is not eligible for surgery, rigid bronchoscopy, or silicone stent implantation due to these potentially hazardous complications. Despite the fact, studies [10,15] also concluded that the SEMS are useful management options for central airway obstruction symptoms in patients with benign and malignant diseases. The complications of this management modality in patients with benign obstructing lesions after placement of SEMSs remain the important issues to be concerned.

The goal of this study was to describe the baseline 
characteristics, clinical features, overall symptomatic response and complication rates after placement of SEMS in patients with benign airway diseases. The managements of SEMS-related complications were also reported.

\section{PATIENTS AND METHODS}

\subsection{Design}

This investigation was a retrospective study. Informed consents were obtained from all patients or their surrogates prior to the procedure of bronchoscopic SEMS implantation and follow up. Methodology and patient confidentiality were approved by our institutional review board (IRB No. 98-3287B). The IRB was also asked to review the design of the project in December 2006 and approved this retrospective study in March 2007.

\subsection{Patients}

From August 2001 to March 2008, 80 patients (mean age $62.5+14.8$ years) with benign airway diseases underwent 124 endoscopic airway stents placement at Chang Gung Memorial Hospital, a university-affiliated hospital in Taiwan. Thoracic surgeons were routinely consulted for feasibility of surgical intervention or rigid bronchoscopy in all patient before SEMS implantation. If patients were unsuitable for surgical intervention due to poor lung function, co-morbidities or they refused surgery, SEMS implantation under fiberoptic bronchoscopy was used if no other treatment options were available. The development of new or progressive symptoms was closely monitored and follow-up radiographic and bronchoscopic examinations were arranged.

\subsection{Stent Implantation}

Ultraflex (Boston Scientific, Natick, MA), a tightly woven, self-expandable metallic stent composed entirely of a single strand of nickel-titanium alloy, was the stent of choice for this study. The principles of SEMS implantation in our institution under conscious sedation and local anesthesia, and the assessment of stent condition were reported in previous studies [16-18]. The choice of stent length and type (with or without cover) was determined by previous endoscopic examination and chest CT scan.

\subsection{Assessment of Stent Condition}

After placement of the stent, a follow-up bronchoscopy was performed in 48 hours. The presence of incomplete stent expansion or incomplete stented airway lumen were recorded. Therefore, those post-procedure factors could be evaluated by the follow-up bronchoscopic studies. In addition, each patient underwent bronchoscopic examination one week after implantation and then every 3 - 6 months thereafter to evaluate stent position and degradation, granulation tissue formation and airway alignment. If new or progressive symptoms including dyspnea, severe cough, increased mucous production or other symptoms that suggest stent fracture occurred, additional bronchoscopy was done.

\subsection{Definition of Complications Related to SEMS}

All the possible complications related to SEMS were confirmed by bronchoscopic examination. According to patients' records, complications in our study included stent migration, granuloma formation, stent fracture, and pneumothorax. Successful management defined as the relief of complications after management without the need for additional procedures during the follow-up period. Five patients with SEMS migration did not require further management as they did not have significant symptoms. The 5 patients were considered as successful management but included in the analysis of stent migration.

\subsection{Statistical Analysis}

Data are expressed as either group percentages (categorical variables) or mean \pm SD (continuous variables). Time variables were expressed as median and Interquartile Range (IQR). All analysis used GraphPad Prism 3.0 (GraphPad Software, San Diego, Calif).

\section{RESULTS}

From August 2001 to March 2008, 80 patients (mean age + standard deviation, $62.5+14.8$ years) with benign tracheobronchial disease received 124 Ultraflex SEMSs. The indications of SEMS implantation are listed in Table 1.

Table 1. Our experience with SEMS.

\begin{tabular}{ccc}
\hline Conditions & Patients numbers & SEMS numbers \\
\hline Malacia & 30 & 65 \\
Post-intubation stenosis & 10 & 12 \\
Post-TB stenosis & 10 & 12 \\
Granulation restenosis & 12 & 16 \\
Stent fracture & 8 & 8 \\
Goiter & 4 & 4 \\
Corrosive injury & 2 & 3 \\
Mediastinitis & 2 & 2 \\
Tracheitis & 2 & 2 \\
Total & 80 & 124 \\
\hline
\end{tabular}

Abbreviations: SEMS: Self-Expandable Metallic Stent; TB: Tuberculosis. 
The demographics of the patients who had placement of SEMSs are listed in Table 2. The baseline characteristics of patients received SEMS implantation including age (mean + standard deviation: $62.5+14.8$ years), gender (male, $60.5 \%)$, smoking (46.8\%), forced expiratory volume in first one second (FEV1) (mean + standard deviation, $0.92+0.41 \mathrm{~L} / \mathrm{s}$ ), follow-up days after SEMS implantation (median (interquartile range); 457 (131 897)) and covered SEMS (33.9\%) were listed. Symptoms improved after SEMS implantation among 95 patients (76.6\%). The clinical presentation in patients with benign airway obstruction included dyspnea (96\%), cough (1.6\%) and respiratory failure $(2.4 \%)$.

The clinical response and complications after placement of SEMS in patients are listed in Table 3. Symptoms improved after SEMS implantation among 95 patients (76.6\%). Time to complications developed was 236 (median; interquartile range, 59 - 672) days. The overall complication rate after SEMS implantation in patients with benign conditions was $41.9 \%$. The 30 days complications rate and the 60 days complications rate were $4.0 \%$ and $8.1 \%$, respectively. The complication episode/patient/month was 0.008 .

Table 4 summarizes the complications rates and the time to detect complications after SEMS implantation. The complication rates included stent migration (6.5\%), granuloma formation (19.3\%) and stent fracture (16.1\%) after SEMS implantation. The time to detect granuloma formation after SEMS implantation in patients with benign airway obstruction was 212 (59 - 489) (median, (IQR) days. The time to detect SEMS migration and fracture were 45 (IQR, 9 - 109) and 686 (IQR, 277 - 856) days, respectively.

Table 2. Demographics of patients with SEMS placement.

\begin{tabular}{lc}
\hline & SEMS (n = 124) \\
\hline Demography & $62.5 \pm 14.8$ \\
Age & $75(60.5)$ \\
Gender, Male, n (\%) & $58(46.8)$ \\
Smoking, n (\%) & $0.92 \pm 0.41$ \\
FEV1 (L/s) & $457(131-897)$ \\
SEMS follow up days, median (IQR) & $42(33.9)$ \\
Cover SEMS, n (\%) & \\
Clinical manifestation before SEMS implantation & $119(96.0)$ \\
Dyspnea, n (\%) & $2(1.6)$ \\
Cough, n (\%) & $3(2.4)$ \\
\hline Respiratory failure, n (\%)
\end{tabular}

Abbreviations: SEMS: Self-Expandable Metallic Stent, FEV1: Forced Expiratory Flow in One Second, FVC: Forced Vital Capacity, IQR: Interquartile Range.
Table 3. Summary of response after SEMS implantation.

\begin{tabular}{lc}
\hline \multicolumn{1}{c}{ Response } & SMES ( $\mathrm{n}=124)$ \\
\hline Resolution of symptom, $\mathrm{n}(\%)$ & $95(76.6)$ \\
Complication related to SEMS in 30 days, $\mathrm{n}(\%)$ & $5(4.0)$ \\
Complication related to SEMS in 60 days, $\mathrm{n}(\%)$ & $10(8.1)$ \\
Overall complications related to SEMS, n (\%) & $52(41.9)$ \\
Time to complications developed. Median (IQR) & $236(59-672)$ \\
Complication episode/patient/month & 0.008 \\
\hline
\end{tabular}

Abbreviations: SEMS: Self-Expandable Metallic Stent; IQR: Interquartile Range.

Table 4. Complications rate and time to detect complications after SEMS placement.

SEMS $(n=124)$

\begin{tabular}{lc}
\hline Complication rate & \\
Stent migration, n (\%) & $8(6.5)$ \\
Granulation tissue formation, n (\%) & $24(19.3)$ \\
Stent fracture, n (\%) & $20(16.1)$ \\
Time to detect complication after SEMS & \\
implantation (days) & \\
Stent migration & $45(9-109)$ \\
Granulation tissue formation & $212(59-489)$ \\
Stent fracture & $686(277-856)$ \\
\hline
\end{tabular}

Abbreviations: SEMS: Self-Expandable Metallic Stent.

The management and outcomes of complications after SEMS placement are listed in Table 5. In patients with stent migration, reposition ( $\mathrm{n}=4,50 \%)$, another stent placement $(\mathrm{n}=3,37.5 \%)$, and stent removal $(\mathrm{n}=1$, $12.5 \%)$ were the measures used to manage this complication. Granuloma formation related to SEMS placement $(n=24)$ was managed by electrocautery $(n=10,41.7 \%)$, balloon dilatation $(\mathrm{n}=1,4.2 \%)$, stent removal $(\mathrm{n}=8$, $33.3 \%)$, or another stent implantation ( $n=5,20.8 \%)$. Stent fracture $(n=20)$ was managed by removal of the fractured stent $(n=10,50 \%)$ or implantation of another stent $(\mathrm{n}=5,25 \%)$. There were 5 fractured stents without any intervention (rate, 25\%) due to minimal protrusion of the stent with patent lumen and preserved architecture. Successful management of SEMS migration, granuloma formation, and SEMS fracture were 100\%, 83.3\%, and $85 \%$, respectively.

\section{DISCUSSION}

In patients with benign airway obstruction not willing to surgery, or medically inoperable, airway stenting may be the only possible treatment [19]. Silicone stents remain the first choice in benign airway obstruction except in patients are unsuitable. In such patients, SEMS are generally indicated $[20,21]$. Our study demonstrated that 
Table 5. Management and outcomes of SEMS-related complications.

\begin{tabular}{cccc}
\hline Complication & Management & Total n (\%) & Successful management, n (\%) \\
\hline Migration & Reposition & & $4(50 \%)$ \\
& Another SEMS stenting & $3(50 \%)$ & $3(37.5 \%)$ \\
Granulation tissue formation & SEMS removal & $1(12.5 \%)$ & $1(12.5 \%)$ \\
& Subtotal & $8(100 \%)$ & $8(100 \%)$ \\
& Electrocautery only & $10(41.7 \%)$ & $8(33.3 \%)$ \\
& Ballon dilatation & $1(4.2 \%)$ & $1(4.2 \%)$ \\
& SEMS removal & $8(33.3 \%)$ & $7(29.1 \%)$ \\
Stent fracture & Another SEMS stenting & $5(20.8 \%)$ & $4(16.7 \%)$ \\
& Subtotal & $24(100 \%)$ & $20(83.3 \%)$ \\
& & & $5(25 \%)$ \\
& None & $5(25 \%)$ & $9(45 \%)$ \\
& SEMS removal & $10(50 \%)$ & $3(15 \%)$ \\
& Another SEMS stenting & $5(25 \%)$ & $18(85 \%)$ \\
\hline
\end{tabular}

Abbreviations: SEMS: Self-Expandable Metallic Stent.

patients with benign airway obstruction who had placement of SEMS to relieve symptoms had poor lung function and were old. Most of our patients with benign airway obstruction presented with dyspnea other than cough and/or respiratory failure. We observed the complication rate in patients with benign airway obstruction. Specifically, granuloma formation and SEMS fracture were not uncommon in patients with benign airway obstruction. This was probably related to the longer period of time the stent was present in the airways of patients with benign airway obstruction and exposure of the stent to the natural and probably excessive compression-decompression cycles of the airway wall during breathing and cough (stress fracture). Most of the complications were managed well and safely by experienced bronchoscopists.

In our institute, surgical treatment in patients with such benign airway diseases is the first choice. If patients were unsuitable for surgical intervention because of poor lung function, comorbidities, or because they refuse surgery, then conservative management and close monitoring will be advised. SEMS placement is considered when patients present with severe symptoms that affect their life quality. The high incidence of dyspnea at the time of presentation in patients with benign airway obstruction may be explained by the worse lung function and older age. The presenting symptoms resolved in $3 / 4$ patients with benign airway obstruction after placement of the SEMS. This is probably related to the isolated involvement of the central airways in patients with benign airway obstruction.

Granulation tissue formation and fractures of SEMS are not rare in patients with benign airway obstruction.
However, complications rates were similar in patients with malignant airway obstruction than patients with benign airway obstruction after 30 and 60 days of follow-up evaluation in previous report [10]. On the other hand, patients with malignancy-induced central airway obstruction may develop certain complications like tumor progression after placement of SEMS. Relationship of this observation to underlying malignancy is possible.

The overall complications rates in our study are similar to previous studies $[10,16]$. SEMS related granuloma formation and stenosis can be managed with a variety of flexible bronchoscopic interventions including electrocautery, cryotherapy, laser photocoagulation, radiofrequency ablation and stent removal (when necessary). In our report, management of SEMS-related complications was feasible and the success rate was more than $80 \%$. However, such interventions for SEMS-related complications require experienced bronchoscopists who are familiar with techniques like electrocautery, balloon dilation, cryotherapy and other interventional pulmonary procedures. Patients who require placement of SEMS must be monitored closely for related complications.

In conclusions, SEMS could be placed under flexible bronchoscopy and conscious sedation with minimal immediate procedure-related complications. Our experience confirms that patients received SEMS implantation due to benign conditions had poor lung function and were old. The complication rate in those patients with benign conditions was high after longer follow-up period. However, interventional pulmonary techniques can be utilized with reasonable success to palliate some SEMS related complacations. 


\section{ACKNOWLEDGEMENTS}

This study was partial supported by grants from Change Gung Memorial Hospital (CMRPG391211 by Drs. F.T.C., C.T.Y., and C.S.K.; CMRPG3B0981-3 by Dr. F.T.C. and CMRPG3B0831-3 by Dr. F.T.C.).

\section{REFERENCES}

[1] Seijo, L.M. and Sterman, D.H. (2001) Interventional pulmonology. The New England Journal of Medicine, 344, 740-749. doi:10.1056/NEJM200103083441007

[2] Bolliger, C.T., Mathur, P.N., Beamis, J.F., et al. (2002) ERS/ATS statement on interventional pulmonology. European Respiratory Society/American Thoracic Society. European Respiratory Journal, 19, 356-373.

[3] Brichet, A., Verkindre, C., Dupont, J., et al. (1999) Multidisciplinary approach to management of postintubation tracheal stenoses. European Respiratory Journal, 13, 888893. doi:10.1034/j.1399-3003.1999.13d32.x

[4] Al-Kattan, K., Simonds, A., Chung, K.F. and Kaplan, D.K. (1997) Kyphoscoliosis and bronchial torsion. Chest, 111, 1134-1137. doi:10.1378/chest.111.4.1134

[5] Colt, H.G. and Harrell, J.H. (1997) Therapeutic rigid bronchoscopy allows level of care changes in patients with acute respiratory failure from central airways obstruction. Chest, 112, 202-206. doi:10.1378/chest.112.1.202

[6] Shaffer, J.P. and Allen, J.N. (1998) The use of expandable metal stents to facilitate extubation in patients with large airway obstruction. Chest, 114, 1378-1382. doi:10.1378/chest.114.5.1378

[7] Noppen, M., Stratakos, G., Amjadi, K., et al. (2007) Stenting allows weaning and extubation in ventilator- or tracheostomy dependency secondary to benign airway disease. Respiratory Medicine, 101, 139-145. doi:10.1016/j.rmed.2006.03.037

[8] Hautmann, H., Bauer, M., Pfeifer, K.J. and Huber, R.M. (2000) Flexible bronchoscopy: A safe method for metal stent implantation in bronchial disease. The Annals of Thoracic Surgery, 69, 398-401. doi:10.1016/S0003-4975(99)01398-3

[9] Madden, B.P., Datta, S. and Charokopos, N. (2002) Experience with Ultraflex expandable metallic stents in the management of endobronchial pathology. The Annals of Thoracic Surgery, 73, 938-944. doi:10.1016/S0003-4975(01)03460-9

[10] Chung, F.T., Chen, H.C., Chou, C.L., et al. (2011) An outcome analysis of self-expandable metallic stents in central airway obstruction: A cohort study. Journal of
Cardiothoracic Surgery, 6, 46. doi:10.1186/1749-8090-6-46

[11] Madden, B.P., Loke, T.K. and Sheth, A.C. (2006) Do expandable metallic airway stents have a role in the management of patients with benign tracheobronchial disease? The Annals of Thoracic Surgery, 82, 274-278. doi:10.1016/j.athoracsur.2006.02.028

[12] Zakaluzny, S.A., Lane, J.D. and Mair, E.A. (2003) Complications of tracheobronchial airway stents. Otolaryngology-Head and Neck Surgery, 128, 478-488. doi:10.1016/S0194-5998(03)00002-0

[13] Gaissert, H.A., Grillo, H.C., Wright, C.D., et al. (2003) Complication of benign tracheobronchial strictures by self-expanding metal stents. The Journal of Thoracic and Cardiovascular Surgery, 126, 744-747. doi:10.1016/S0022-5223(03)00361-1

[14] Noppen, M., Meysman, M., Claes, I., D’Haese, J., et al. (1999) Screw-thread vs Dumon endoprosthesis in the management of tracheal stenosis. Chest, 115, 532-535. doi:10.1378/chest.115.2.532

[15] Husain, S.A., Finch, D., Ahmed, M., et al. (2007) Longterm follow-up of ultraflex metallic stents in benign and malignant central airway obstruction. The Annals of Thoracic Surgery, 83, 1251-1256. doi:10.1016/j.athoracsur.2006.11.066

[16] Chung, F.T., Lin, S.M., Chen, H.C., et al. (2010) Factors to obstructive grangulation tissue formation after ultraflex stent implantation in benign tracheal stenosis. The Journal of Thoracic and Cardiovascular Surgery, 58, 102107. doi:10.1055/s-0029-1186266

[17] Chung, F.T., Chen, G.Y., Chou, C.L., et al. (2012) Remove airway ultraflex stents by flexible bronchoscope study. The American Journal of the Medical Sciences, 343, 267-272. doi:10.1097/MAJ.0b013e31822a6bc3

[18] Chung, F.T., Lin, H.C., Chou, C.L., et al. (2012) Airway ultraflex stenting in esophagorespiratory fistula caused by esophageal cancer. The American Journal of the Medical Sciences, 344, 105-109. doi:10.1097/MAJ.0b013e3182367b6a

[19] Wood, D.E., Liu, Y.H., Vallieres, E., et al. (2003) Airway stenting for malignant and benign tracheobronchial stenosis. The Annals of Thoracic Surgery, 76, 167-172. doi:10.1016/S0003-4975(03)00033-X

[20] Noppen, M., Stratakos, G., D’Haese, J., et al. (2005) Removal of covered self-expandable metallic airway stents in benign disorders: Indications, technique, and outcomes. Chest, 127, 482-487. doi:10.1378/chest.127.2.482

[21] Nesbitt, J.C. and Carrasco, H. (1996) Expandable stents. Chest Surgery Clinics of North America, 6, 305-328. 